\title{
Der Einfluss städtischer Grünflächen auf die Immobilienpreise: Eine hedonische Analyse für die Stadt Berlin
}

\author{
Henry Wüstemann ${ }^{1} \cdot$ Jens Kolbe $^{2}$
}

Eingegangen: 22. März 2016 / Angenommen: 28. Februar 2017 / Online publiziert: 10. März 2017

(C) Springer-Verlag Berlin Heidelberg 2017

Zusammenfassung Grünflächen und Grünstrukturen stellen eine wichtige Grundlage für die nachhaltige Entwicklung von Städten dar. Der Nutzen von städtischen Grünstrukturen kann sich auch in höheren Immobilien- und Grundstückspreisen widerspiegeln. Gibt es auf internationaler Ebene eine Vielzahl empirischer Studien, die den Einfluss städtischer Freiflächen auf den Wert von Immobilien belegen, so sind es in Deutschland vergleichsweise wenige. Ziel der vorliegenden Studie ist eine Analyse des Einflusses von urbanen Grünflächen auf die Immobilienpreise in Deutschland. Dazu wurden Daten von Einfamilienhausverkäufen der Jahre 1996-2012 der Kaufpreissammlung des Gutachterausschusses für Grundstückswerte (GAA) für die Stadt Berlin sowie georeferenzierte Daten des European Urban Atlas (EUA) zur Grünausstattung verwendet. Zudem wurde kontrolliert, inwieweit sich andere Landnutzungen wie Wasser, Wald und Brachflächen im Vergleich zu Grünflächen auf die Immobilienpreise auswirken. Die Analysen zeigen einen positiven Einfluss von städtischen Parkanlagen auf die Immobilienpreise. Die Ergebnisse liefern wichtige Informationen zum Umfang der Kapitalisierung von urbanen Grünflächen in den Immobilienpreisen.

Henry Wüstemann

henry.wuestemann@tu-berlin.de

Jens Kolbe

j.kolbe@tu-berlin.de

1 Fachgebiet Landschaftsökonomie, Technische Universität Berlin, Straße des 17. Juni 145, 10623 Berlin, Deutschland

2 Fachgebiet Ökonometrie und Wirtschaftsstatistik, Technische Universität Berlin, Straße des 17. Juni 135, 10623 Berlin, Deutschland
Schlüsselwörter Urbanes Grün · Immobilienwertmethode · GIS-Analyse · Stadtentwicklung · Ökosystemleistungen

The impact of urban green space on real estate prices: A hedonic analysis for the city of Berlin

Abstract Urban green space represents a key aspect for the sustainable development of urban areas. The benefit of urban green space can also be reflected in higher property and land prices. Although a high number of international studies prove the impact of urban green on real estate prices, the empirical basis in Germany is still very limited. The overall purpose of this study is to investigate the impact of urban green space on real estate prices in Germany. The data set contains transaction data for single family homes (1996-2012) provided by the Committee of Valuation Experts (GAA, Gutachterausschuss für Grundstückswerte) for the city of Berlin and cross section geo-coded data for urban green drawn from the European Urban Atlas (EUA) of the European Environment Agency. In order to control for additional open space categories we further incorporated geo-coded data on water bodies, forest, farmland and fallow land. The analysis shows a positive impact of urban green on real estate prices. The results provide helpful information on the capitalization of urban green space in real estate prices.

Keywords Urban green space · Hedonic pricing - GISAnalysis · Urban development $\cdot$ Ecosystem services 


\section{Einleitung}

Grünflächen und Grünstrukturen in Städten steigern das menschliche Wohlbefinden in hohem Maße und auf vielfältige Weise. Für die nachhaltige Entwicklung von Städten sind sie unentbehrlich. Sie stehen für eine Fülle positiver Effekte auf den Klima- und Biodiversitätsschutz, die Luftqualität und die Erholung (Rowntree/Nowak 1991; Kuhn/Brandl/Klotz 2004; Nowak/Crane/Stevens 2006). Folgerichtig werden in der „Nationalen Strategie zur biologischen Vielfalt" der Bundesregierung eine deutliche Erhöhung des Anteils grüner Flächen und Strukturen, ihre Vernetzung und eine qualifizierte Innenentwicklung von Siedlungen sowie eine verminderte Flächeninanspruchnahme gefordert (BMUB 2007). Um eine naturverträgliche Stadtentwicklung sicherzustellen, sollte bei stadtplanerischen Entscheidungen neben bestehenden Planwerken auch die ökonomische Bedeutung von urbanen grünen Infrastrukturen berücksichtigt werden.

Der Nutzen von städtischen Grünstrukturen können sich auch in höheren Immobilien- und Grundstückpreisen widerspiegeln. Viele Studien auf internationaler Ebene haben in diesem Zusammenhang den Einfluss städtischer Grünflächen auf den Wert von Immobilien mittels der Immobilienwertmethode (Hedonische Preismethode) nachgewiesen (u.a. Acharya/Bennett 2001; Irwin 2002; Morancho 2003; Kong/Yin/Nakagoshi 2007). Die Immobilienwertmethode basiert auf der Annahme, dass sich ein Gut durch viele Eigenschaften beschreiben lassen kann und der Wert der Immobilien durch verschiedene strukturelle (intrinsische) Variablen wie Größe und Alter der Immobilie sowie extrinsische Variablen wie die Lage der Immobilie bestimmt wird. Im Rahmen der extrinsischen Faktoren können auch spezifische Umweltfaktoren wie die Lärm- und Feinstaubbelastung oder aber die Verfügbarkeit von urbanen Grünflächen Auswirkungen auf die Preise von Immobilien haben.

In den letzten Jahren wurde eine nennenswerte Anzahl Artikel veröffentlicht, die verschiedene hedonische Analysen des deutschen Wohnungsmarktes zum Thema haben (Ahlfeldt/Maennig 2010a; Ahlfeldt/Maennig 2010b; Kholodilin/Mense 2012; Promann 2012; Brandt/Maennig/ Richter 2013; Mense/Kholodilin 2014). Ziel der vorliegenden Untersuchung ist die Analyse des Einflusses von urbanen Grünflächen auf die Immobilienpreise mittels der Immobilienwertmethode. Dazu wurden im Rahmen eines durch das Bundesamt für Naturschutz $(\mathrm{BfN})$ geförderten Projektes ${ }^{1}$ Daten der Kaufpreissammlung des Gutachterausschusses für Grundstückswerte (GAA) ${ }^{2}$ für die Stadt Berlin mit georeferenzierten Daten zur Grünausstattung des Urban Atlas der European Environment Agency (EEA)

\footnotetext{
${ }^{1}$ FKZ: 3512821400.

2 Vgl. http://www.berlin.de/gutachterausschuss/ (12.01.2017).
}

analysiert und der Einfluss von urbanen Grünflächen auf die Preisbildung von Immobilien systematisch untersucht. In Kapitel 2 dieses Beitrags erfolgt eine Analyse bisheriger Studien zur Bewertung von urbanen Grünflächen mittels hedonischer Analyse. In Kapitel 3 werden die Datenbasis und das methodische Vorgehen beschrieben und in Kapitel 4 die Ergebnisse der Untersuchung dargestellt. Kapitel 5 enthält ein Fazit sowie einen Ausblick.

\section{Die Bewertung von urbanem Grün mittels Immobilienwertmethode}

Die Immobilienwertmethode basiert grundlegend auf der Annahme, dass eine Immobilie ein heterogenes Gut ist, dessen Preis durch eine Vielzahl von intrinsischen und extrinsischen Variablen bestimmt wird und sich wie folgt beschreiben lässt:

$P=f\left(S_{1} \ldots S_{k}, N_{1} \ldots N_{m}, Z_{1} \ldots Z_{n}\right)$

Dabei steht $S$ für die strukturellen oder intrinsischen Charakteristika (Größe des Hauses, Anzahl der Zimmer, Alter etc.), $N$ bezeichnet geographische Variablen wie die Anzahl der Schulen oder die Qualität des öffentlichen Nahverkehrs und $Z$ repräsentiert spezifische Umweltqualitäten (Luftqualität, Lärm) oder aber die Lagebeziehung zu spezifischen Landschaften bzw. Flächen (Bateman 1993: 55; Morancho 2003: 36). Nach Bateman (1993: 54 f.) fußt die hedonische Preismethode auf verschiedenen Prinzipien, wie zum Beispiel der Annahme, dass die Zahlungsbereitschaft eine angemessene Einheit ist, um den Nutzen abzubilden. Eine weitere Grundannahme ist, dass Individuen in der Lage sind, Veränderung von Umweltqualität wahrzunehmen und diese Veränderungen einen Einfluss auf den Nutzen, den Immobilien stiften, haben. Weiterhin wird angenommen, dass die gesamte Studienregion einen vollständigen Markt darstellt, in dem alle Informationen bezüglich der Hauspreise und Umweltcharakteristika vorliegen und der sich zudem in einem Gleichgewicht befindet. Darüber hinaus besteht die Annahme, dass der Nutzen aus dem urbanen Grün über die Zeit konstant ist.

Bei der Betrachtung dieser Annahmen wird schnell klar, dass sie sehr restriktiv sind und so in der Realität nur selten vorliegen. Ein Kaufinteressent wird sicher selten über alle Informationen bezüglich bestehender Hauspreise, zukünftige Entwicklungen sowie aller preisbildenden Faktoren (z. B. urbanes Grün) verfügen. Auch ist nur schwer vorstellbar, dass sich ein Immobilienmarkt in einem absoluten Gleichgewicht befindet, da Wohnungsmärkte in der Regel sehr unflexibel reagieren. Kommt es beispielsweise zu einem rasanten Anstieg der Bevölkerung in einer Stadt, kann der plötzlich gestiegenen Nachfrage nur stark zeitver- 
zögert durch verstärkte Investitionen in den Wohnungsbau begegnet werden.

Die Bewertung von urbanen Frei- und Grünflächen mittels der Immobilienwertmethode hat eine lange Tradition in der wissenschaftlichen Literatur (u.a. Kitchen/Hendon 1967; Acharya/Bennett 2001; Lutzenhiser/Netusil 2001; Irwin 2002; Morancho 2003; Melichar/Vojáček/Rieger et al. 2009). Ergebnisse dieser Bewertungsstudien weisen dabei häufig eine Kapitalisierung von urbanem Grün in Haus- und Grundstückspreisen nach. In diesem Zusammenhang konnte zudem gezeigt werden, dass Qualitäten von Grünflächen wie die Größe und Naturnähe auch einen Einfluss auf den Preis von Immobilien ausüben können (Lutzenhiser/Netusil 2001; Morancho 2003; Panduro/Lausted Veie 2013).

Morancho (2003) analysiert basierend auf einer Stichprobe von 810 Beobachtungen den Einfluss von städtischem Grün auf die Immobilienpreise in Castellón (Spanien) und betrachtet als Lagevariablen die Existenz von Parks oder öffentlichen Gärten, die Distanz der Immobilie zur nächstgelegenen Grünfläche und die Größe dieser Fläche. Morancho (2003: 38) zeigt, dass strukturelle Variablen den stärksten Einfluss innerhalb der Preisfunktion ausüben und eine negative Korrelation zwischen dem Immobilienpreis und der Distanz zur nächstgelegenen Grünfläche existiert. Melichar, Vojáček, Rieger et al. (2009: 16) verwenden einen Datensatz von 1.701 Transaktionen in Prag (Tschechien) für den Zeitraum von 2005 bis 2008. Ihre Ergebnisse zeigen, dass die Wohnungsgröße den größten Einfluss innerhalb der Preisfunktion auf den Kaufpreis der Immobilie ausübt (Melichar/Vojáček/Rieger et al. 2009: 19). Zudem konnte eine inverse Beziehung zwischen der Distanz zur nächstgelegenen Waldfläche und dem Immobilienpreis nachgewiesen werden, was darauf hindeutet, dass eine höhere Zahlungsbereitschaft für solche Immobilien existiert, die näher an Waldflächen gelegen sind. Panduro und Lausted Veie (2013: 121) analysieren den Einfluss von acht verschiedenen Grünflächenkategorien auf die Preise von Immobilien in Aalborg (Dänemark) und nutzen dafür insgesamt 12.928 Immobilientransaktionen im Zeitraum von 2000 bis 2007. Als Ergebnis ihrer Analysen halten Panduro und Lausted Veie (2013: 126) fest, dass insbesondere solche Grünflächen einen Einfluss auf den Immobilienpreis ausüben, die einen guten Zugang und einen guten Erhaltungszustand aufweisen. Lutzenhiser und Netusil (2001: 293) zeigen anhand einer Analyse von Hauspreisen in Portland (Oregon) zwischen 1990 und 1992, dass Hauspreise in der Nähe von städtischen Parks vergleichsweise niedriger sind, liegen die Immobilien jedoch näher an Parks mit natürlicher Vegetation, steigen deren Preise. Zudem können sie einen positiven Zusammenhang zwischen der Größe städtischer Parks und den Kaufpreisen für Immobilien nachweisen (Lutzenhiser/ Netusil 2001: 295).
Die bisher vorliegenden Studien zum Einfluss von Stadtgrün auf die Preise von Immobilien geben auch einen Hinweis auf die Höhe der Kapitalisierung von urbanem Grün in den Immobilienpreisen. So zeigt eine Untersuchung zum Einfluss von Stadtgrün auf die Immobilienpreise am Beispiel von 590 Reihenhäusern in der Stadt Salo (Finnland), dass ein 1.000m-Anstieg der Distanz zu Waldflächen zu durchschnittlichen Preisabschlägen bei den Hauspreisen von 5,9\% führen würde (Tyrväinen/Miettinen 2000: 215). Morancho (2003: 39) zeigt in der bereits erwähnten Studie für Castellón (Spanien), dass ein 100m-Anstieg der Distanz zum nächstgelegenen Park zu mittleren Preisabschlägen bei den Immobilien von 1.800 Euro führen würde. Kong, Yin und Nakagoshi (2007: 249) weisen für einen Anstieg der Grün-Abdeckung im 300m-Umkreis um die Immobilie in Jinan (China) einen durchschnittlichen Preisanstieg von $2,1 \%$ nach.

Aufgrund der analysierten Studien bleibt festzuhalten, dass intrinsische Faktoren wie die Größe der Wohnung und die Anzahl der Zimmer einen wesentlich stärkeren Einfluss auf den Kaufpreis von Immobilien ausüben als extrinsische Faktoren wie die Lage zu Bildungseinrichtungen, Infrastruktur und eben auch städtische Grünflächen.

In Deutschland gibt es bisher vergleichsweise wenige Anwendungen der hedonischen Preismethode zur Bewertung von städtischem Grün (u. a. Promann 2012). Ein Grund dafür könnte sein, dass die deutsche Wertermittlungspraxis durch große Beurteilungsspielräume für Sachverständige, univariate Verfahren oder die Tendenz zur Arbeit mit Einzel- und Vergleichsfällen (vgl. z. B. § 558a Abs. 2 BGB oder der sogenannte Tabellenmietspiegel) geprägt ist. Bei den Erstellern von Regressionsmietspiegeln liegen zwar sowohl Daten als auch wissenschaftlich qualifizierte Auswertungen vor, deren Erkenntnisse werden jedoch vor dem Hintergrund der Anwendung in mietrechtlichen Auseinandersetzungen selten publiziert (Wüstemann/Kolbe/von Malottki et al. 2016).

\section{Methodisches Vorgehen}

\subsection{Datenbasis}

Im Mittelpunkt der Untersuchung steht die Analyse des Einflusses von städtischen Grünstrukturen auf die Immobilienpreise mittels der Immobilienwertmethode. Dazu werden Daten der Kaufpreissammlung des Gutachterausschusses für Grundstückswerte (GAA) von Berlin verwendet. Der Gutachterausschuss führt nach $\S 193$ Abs. 3 BauGB eine Kaufpreissammlung und wertet diese systematisch aus. Der für die Analysen verwendete Datensatz umfasst Verkäufe von 26.196 Einfamilienhäusern für die Stadt Berlin (Zeitraum: 1996-2012). Prinzipiell werden hier alle vom Berliner 
Abbildung 1 Preisentwicklung der Berliner Einfamilienhäuser im Zeitraum 19962012

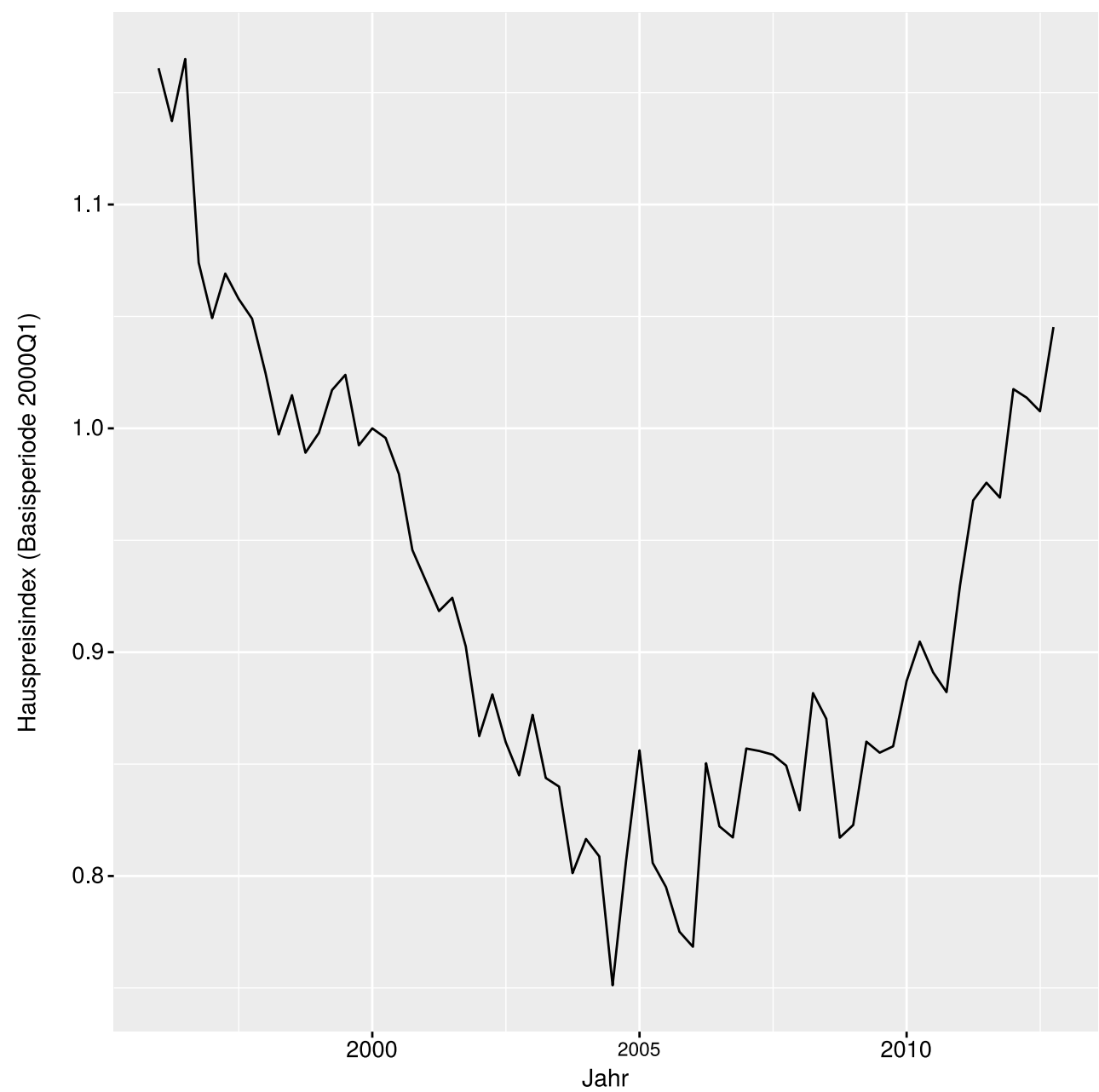

Gutachterausschuss erfassten Transaktionen in die Analysen einbezogen. Allerdings wurden aus dem Datensatz unter anderem paarweise identische Beobachtungen und Beobachtungen mit ungewöhnlich hohen oder niedrigen Kaufpreisen (bezieht sich auf den Preis pro Quadratmeter) entfernt. Auch wurden solche Transaktionen, für die besondere Umstände im Geschäftsverkehr ausgewiesen wurden (z. B. Erbauseinandersetzungen, geschäftliche Bindungen), gelöscht. Zudem wurden Transaktionen extrahiert, für die keine vollständigen Adressdaten bzw. Georeferenzierungen vorhanden waren, da für diese Beobachtungen kein räumlicher Zusammenhang mit den urbanen Grünflächen hergestellt werden kann. Um die zeitliche Vergleichbarkeit der Preise zu gewährleisten, wurden die Hauspreise auf ,Jahr 2000 Preise ' mittels eines hedonischen Hauspreisindex ${ }^{3} \mathrm{zu}-$ rückgerechnet. Abbildung 1 zeigt die Preisentwicklung der

\footnotetext{
3 Vgl. Schulz/Werwatz (2011). Die Bereinigung der Hauspreise basiert auf einem vorher geschätzten hedonischen (qualitätsbereinigten) Hauspreisindex. Dieses Vorgehen ist für Immobilien wie für andere Konsumgüter (z. B. Computer) gebräuchlich (vgl. auch Goh/Costello/ Schwann (2012: 649 f.).
}

Berliner Einfamilienhäuser im Zeitraum 1996-2012. Neben der Georeferenzierung wird jede Beobachtung im Datensatz mittels verschiedener quantitativer und qualitativer Variablen (z. B. Größe der Immobilie, Alter, Geschossanzahl) ausführlich beschrieben (vgl. Tabelle 1).

Um Aussagen zum Einfluss von städtischen Grünflächen auf die Immobilienpreise machen zu können, wird der aufbereitete Datensatz der Kaufpreissammlung mit georeferenzierten Informationen zur urbanen Landnutzung in Berlin des Urban Atlas (Referenzjahr 2006) der Europäischen Umweltagentur verschnitten (EEA 2011). Als Flächennutzung werden die Themenbereiche „Urbanes Grün“, „Brachflächen“, „Wald“, „Wasser" und „Landwirtschaft" betrachtet.

\subsection{Erklärende und abhängige Variablen}

Als abhängige Variable wird entsprechend der Zielstellung der Untersuchung der absolute Kaufpreis der Immobilien angesetzt. Für das Hauspreissample der Stadt Berlin lagen zudem Informationen zu einer Vielzahl von strukturellen Variablen der Einfamilienhäuser (u. a. Geschossfläche, Alter der Immobilie, Grundstücksfläche, Dachform) vor, die 
Tabelle 1 Beispiele für die betrachteten Variablen in der hedonischen Preisfunktion

\section{Variablen}

intrinsisch (strukturell)

extrinsisch
Allgemeine Lagevariablen

Grünflächenbezogene Variablen
Größe der Immobilie, Alter der Immobilie, Grundstücksfläche, Dachform etc. Bezirke, Ortsteile, Wohnlage nach Mietspiegel

Grünflächenanteil im 500m-Umkreis um die Immobilie in \% (bezogen auf die gesamte Kreisfläche im 500m-Radius um die Immobilie) als Kontrollvariablen in der vorliegenden Untersuchung genutzt wurden. Die hier vorgestellten Regressionen beruhen auf Berechnungen mit allen verfügbaren strukturellen Variablen. Da es stärkere Variationen der durchschnittlichen Hauspreise für spezifische Stadtbezirke (z.B. aufgrund der unterschiedlichen Attraktivität der Bezirke) geben kann, wird in der Untersuchung auch für die jeweiligen Stadtbezirke, Ortsteile und Wohnlagen kontrolliert.

Im Fokus der hedonischen Analyse steht der Einfluss von urbanen Grünflächen auf die Immobilienpreise. Aus diesem Grund kommt der Betrachtung von grünflächenbezogenen Variablen im Rahmen der Untersuchung eine besondere Bedeutung zu. Um abschätzen zu können, welchen Einfluss der Anteil an städtischen Grünflächen auf die Kaufpreise von Immobilien hat, wird eine Variable ,Grünflächenanteil (in \%) in einem 500m-Radius um die Immobilie generiert. Um auch auf den Einfluss anderer urbaner Landnutzungen kontrollieren zu können, wurden auch Flächenanteilsvariablen für „Wasser“, „Landwirtschaft“, „Brachflächen“ und „Wald" berechnet.

\subsection{Das empirische Modell}

Innerhalb der Immobilienwertmethode spielt die Betrachtung der hedonischen Preisfunktion eine zentrale Rolle. Funktionelle Formen zur Schätzung der hedonischen Preisfunktionen beinhalten unter anderem lineare, quadratische, semi-log-, log-log- und sogenannte Box-Cox-Transformationen (Appelbaum 1979: 450 ff.). In der hedonischen Theorie finden sich nur wenige Hinweise bezüglich der
Spezifikation der funktionellen Form in der hedonischen Preisfunktion und die Bestimmung der geeigneten Schätzform erfolgt zumeist empirisch (Vanslembrouck/Van Huylenbroeck/Van Meensel 2005: 24). Viele Autoren bevorzugen zur Schätzung der hedonischen Preisfunktion ein sogenanntes semi-logarithmisches (semi-log) Modell (Malpezzi 2003: 77 ff.). Ein Grund dafür ist die vergleichsweise einfache Interpretation der geschätzten Koeffizienten. Diese lassen sich relativ einfach als prozentuale Preisänderungen bei der Berechnung der impliziten Preise interpretieren. Zur Schätzung der hedonischen Preisfunktion wurde aus den genannten Gründen ein Semi-log-Modell angewendet:

$\ln \left(P_{n}\right)=\beta_{0}+\sum_{c=1}^{C} \beta_{c} x_{c n}+\sum_{d=1}^{D} \gamma_{d} x_{d n}+\epsilon_{n}$

Die Variablen $x_{c n}$ beschreiben die stetigen Eigenschaften der verkauften Immobilien (Fläche, Alter, Geschossfläche, Distanz zum Stadtzentrum, Flächennutzungsindikatoren), $n=1, \ldots, N$ und die Variablen $x_{d n}$ die diskreten Charakteristika (Wohnlage nach Mietspiegel, Kellertyp, Dachform, Ortsteile, Bezirke, Haustyp), die als Dummy-Variablen in die Formel eingehen. Den stetigen Variablen Fläche, Geschossfläche und Alter wird ein nichtlinearer Einfluss auf den Preis unterstellt, weswegen sie in der vorliegenden Untersuchung auch quadriert in die Gleichung eingehen. Weiterhin sind $\beta_{c}$ bzw. $\gamma_{d}$ die Koeffzienten der jeweiligen Eigenschaften in diesem Regressionsmodell und werden, entsprechend der hedonischen Theorie, als implizite Preise interpretiert. Bei den Lagevariablen in unserem Modell

Tabelle 2 Deskriptive Statistik der strukturellen Variablen (Beispiele) und der Landnutzungsvariablen ${ }^{\mathrm{a}}$

\begin{tabular}{llllll}
\hline Variable & Mittelwert & Median & Standardabweichung & Minimum & Maximum \\
Preis (in Euro) & $314.088,20$ & $262.871,8$ & $217.312,90$ & $35.261,85$ & $5.686 .953,00$ \\
Geschossfläche $\left(\right.$ in m $^{2}$ ) & 148,00 & 137,00 & 66,58 & 30,00 & $3.612,00$ \\
Grundstücksfläche (in m ${ }^{2}$ ) & 560,15 & 505,00 & 338,71 & 82,00 & $16.250,00$ \\
Alter (in Jahren) & 41,89 & 41,00 & 30,27 & 0,00 & 211,00 \\
500m-Umkreis um die Immobilie in \% & 5,39 & & & 0,00 \\
Parks & 1,69 & 2,56 & 7,27 & 0,00 & 60,11 \\
Wasser & 4,48 & 0,00 & 5,74 & 0,00 & 7,56 \\
Wald & 5,08 & 0,00 & 10,81 & 0,00 & 73,48 \\
Landwirtschaft & 0,20 & 0,00 & 9,75 & 0,00 & 5,83 \\
Brachflächen & 0,00 & 0,58 & & \\
\hline
\end{tabular}

${ }^{a}$ Es handelt sich um mittels hedonischen Preisindex inflationsbereinigte Preise (vgl. Schulz/Werwatz 2011 sowie Kapitel 3.1).

${ }^{\mathrm{b}}$ Grünflächenanteil im 500m-Umkreis um die Immobilie in \% (bezogen auf die gesamte Kreisfläche im 500m-Radius um die Immobilie) 
Tabelle 3 Regressionsergebnisse der hedonischen Preisfunktion für die Stadt Berlin

\begin{tabular}{ll}
\hline Variable & Koeffizient \\
Grundstücksfläche & $0,00040^{* * *}$ \\
Grundstücksfläche (quadriert) & $-0,00000008$ \\
Alter & $-0,013^{* * *}$ \\
Alter (quadriert) & $0,000076^{* * *}$ \\
Geschossfläche & $0,0029 * * *$ \\
Geschossfläche (quadriert) & $-0,000007^{* * *}$ \\
Charlottenburg-Wilmersdorf & $0,295^{* * *}$ \\
Spandau & $-0,119^{* * *}$ \\
Steglitz-Zehlendorf & $0,439^{* * *}$ \\
Tempelhof-Schöneberg & $-0,031$ \\
Neukölln & $-0,098^{* * *}$ \\
Treptow-Köpenick & $-0,155^{* * *}$ \\
Marzahn-Hellersdorf & $-0,230^{* * *}$ \\
Lichtenberg & $-0,177^{* * *}$ \\
Reinickendorf & $-0,036^{* *}$ \\
Grünflächen & $0,001306^{*}$ \\
Brachflächen & $-0,022^{* *}$ \\
Wald & $-0,000237$ \\
Wasser & $0,0051955^{* * *}$ \\
Landwirtschaft & $-0,0006879$ \\
Konstante & $12,66^{* * *}$ \\
Anzahl der Beobachtungen & 26.196 \\
$\mathrm{R}^{2}$ & $0,71^{\mathrm{a}}$ \\
\hline
\end{tabular}

Die abhängige Variable ist der logarithmierte absolute Kaufpreis; Signifikanzniveaus: *signifikant $(\mathrm{p}<0,05)$; **sehr signifikant $(\mathrm{p}<$ $0,01) ; * *$ hoch signifikant $(\mathrm{p}<0,001)$; nicht signifikant $(\mathrm{p} \geq 0,05)$; Referenzkategorien: Bezirk Pankow.

Insgesamt enthält die letztendliche hedonische Preisfunktion über 100 Kontrollvariablen (Grundstücksfläche, Alter, Ortsteile, Wohnlagen, Dachformen etc.), von denen hier beispielhaft aber nur die Geschossund Grundstücksfläche sowie das Alter der Immobilie aufgeführt werden. Um auch auf einen nichtlinearen Einfluss der Grundstücksund Geschossfläche sowie des Alters der Immobilie kontrollieren zu können, wurden jeweils auch quadrierte Variablen mit in das Modell aufgenommen.

a Bei dem hier angegebenen $R^{2}$ handelt es sich um ein $R^{2}$ aus einer ,Standard-OLS-Regression", welches von der Statistiksoftware Stata ausgegeben wird, aber für unser Modell wegen des clusterrobusten Schätzers nicht korrekt berechnet werden kann.

handelt es sich um die Wohnlage nach dem Mietspiegel, den Bezirk und um den Ortsteil, in dem die jeweilige Beobachtung liegt. Durch die räumliche Gruppierung (Aggregation der Lage auf Ortsteilniveau) der Daten kommt es zu einer verzerrten Berechnung der Standardfehler im Regressionsmodell. Um diesem Effekt entgegenzuwirken, nutzen wir einen clusterrobusten Schätzer der Kovarianzmatrix auf der Aggregationsebene der Ortsteile (vgl. Moulton 1986; Moulton 1990).

\section{Ergebnisse}

Eine deskriptive Statistik der strukturellen Daten sowie der Landnutzungsdaten ist in Tabelle 2 zusammengestellt, die eigentlichen Regressionsergebnisse in Tabelle 3. Da die hedonische Preisfunktion dieser Studie eine Vielzahl von Regressoren ${ }^{4}$ beinhaltet, werden in Tabelle 3 nur die wichtigsten intrinsischen Variablen wie Geschoss, Grundstücksfläche und das Alter der Immobilien sowie die Berliner Bezirke als Lagevariablen wiedergegeben.

Abbildung 2 zeigt die durchschnittlichen Quadratmeterpreise des Hauspreissamples (Preis pro Quadratmeter Geschossfläche) für die Berliner Stadtbezirke. In Abbildung 3 ist hingegen der durchschnittliche Quadratmeterpreis pro Grundstücksfläche der Hauspreisstichprobe für die Stadtbezirke in Berlin dargestellt. Dabei sind Variationen der durchschnittlichen Preise pro Quadratmeter Geschoss- und Grundstücksfläche über die Stadtbezirke zu erkennen, mit besonders hohen Quadratmeterpreisen in den Bezirken Charlottenburg-Wilmersdorf und Steglitz-Zehlendorf sowie besonders niedrigen Preisen im Bezirk Treptow-Köpenick. Für die Bezirke Mitte und Friedrichshain-Kreuzberg waren in der Datenbasis insgesamt 34 Transaktionen von Einfamilienhäusern ausgewiesen. Diese betreffen zum großen Teil besondere Immobilien, die sich vereinzelt in der Nachbarschaft zu Mietshäusern befinden und nicht notwendigerweise Einfamilienhäuser darstellen, weshalb sie in der Analyse und den nachfolgenden Abbildungen nicht berücksichtigt worden sind.

Die Ergebnisse der statistischen Analysen sind in Tabelle 3 dargestellt. Die Koeffizienten in den Spalten geben die Stärke und - je nach Vorzeichen - die Richtung des Einflusses der Variablen auf den Immobilienpreis an. Der Einfluss der strukturellen Variablen entspricht den Erwartungen. So hat die Grundstücks- und Geschossfläche einen positiven Einfluss auf den Immobilienpreis. Das Alter der Immobilie hingegen beeinflusst deren Preis negativ. Ein Anstieg des Alters der Immobilie um ein Jahr würde hier beispielsweise zu durchschnittlichen Preisabschlägen von 0,68\% des Kaufpreises der Immobilie führen. Zudem zeigt die Kontrolle der quadrierten Geschossflächen und des Alters der Immobilie, dass hier ein nichtlinearer Zusammenhang zwischen dem Immobilienpreis und diesen Variablen vorliegt. Bei den Kontrollen für die Lage der Immobilien in den jeweiligen Berliner Stadtbezirken geht - im Vergleich zum Bezirk Pankow - ein positiver Einfluss von den Bezirken Charlottenburg-Wilmersdorf und Steglitz-Zehlendorf aus, ein negativer Einfluss hingegen unter anderem von den Bezirken Neukölln, Spandau und Treptow-Köpenick.

\footnotetext{
${ }^{4}$ Insgesamt enthält die hedonische Preisfunktion dieser Studie 136 Regressoren.
} 
Abbildung 2 Verteilung der Quadratmeter Geschossfläche des Hauspreissamples über die Berliner Stadtbezirke Quelle: Gutachterausschuss für Grundstückswerte Berlin, Zeitraum 1996-2012 durchschnittlichen Preise pro

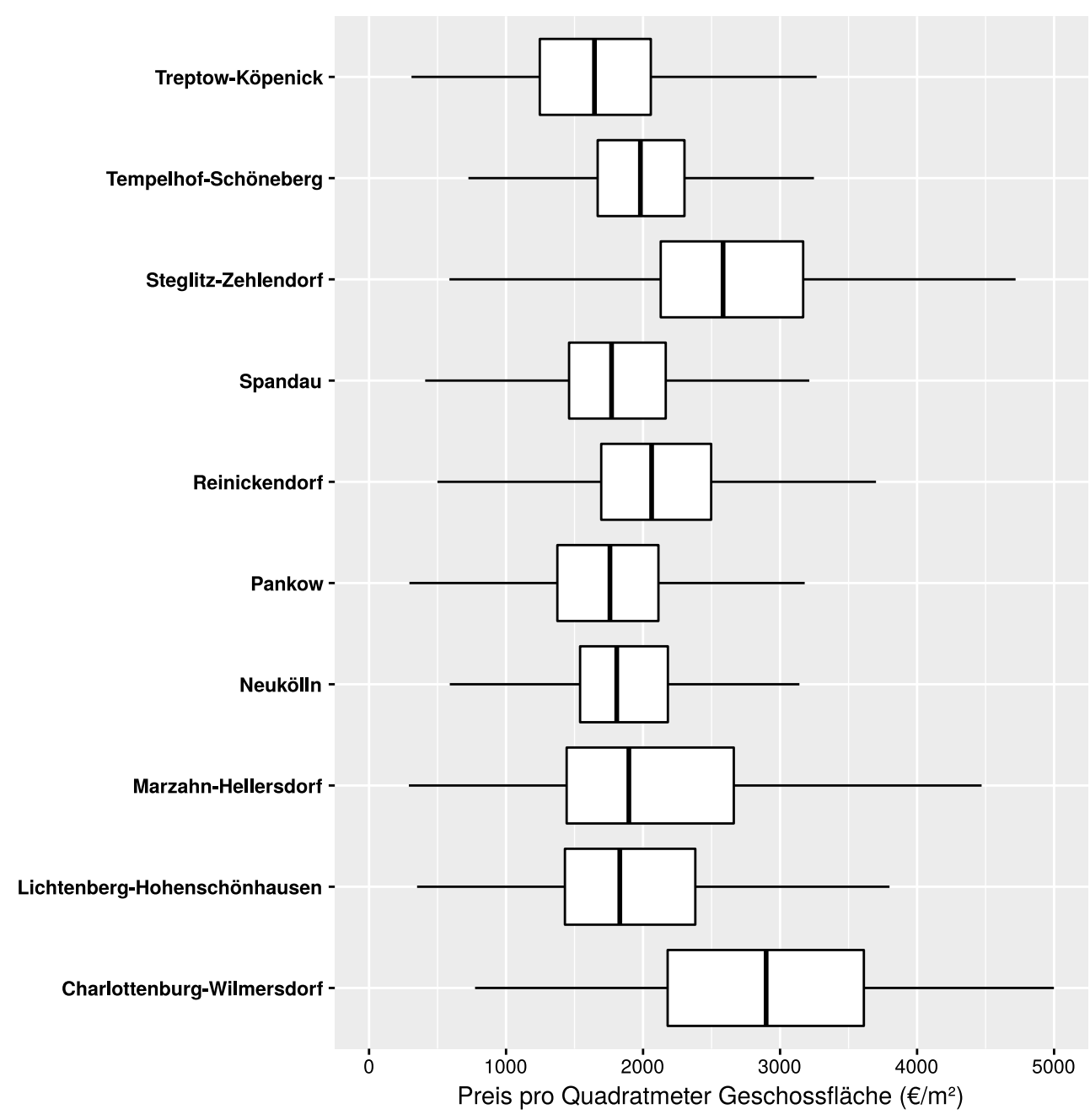

Einen positiven Einfluss der Landnutzung auf den Immobilienpreis bringen Grünflächen sowie Wasserflächen. Eine Erhöhung des Grünflächenanteils im 500m-Umkreis um die Immobilie um $1 \%$ würde hier zu einer Erhöhung der Immobilienpreise um 0,13\% führen. Ein hoher Anteil an Brachflächen in der Nähe der Immobilien führt hingegen zu vergleichsweise deutlichen Preisabschlägen. So würde eine Erhöhung der Abdeckung an Brachflächen im 500m-Umkreis der Immobilie um $1 \%$ Preisabschläge von 2,2 \% des Immobilienpreises zur Folge haben. Für die Flächennutzungen ,Wald' und ,Landwirtschaft' konnten keine signifikanten Ergebnisse nachgewiesen werden. Eine Kontrolle der Variablen für, Wald ' und ,Landwirtschaft ' in einem Regressionsmodell ohne korrigierte Standardfehler zeigt hingegen signifikante Ergebnisse. Dies deutet darauf hin, dass die Beobachtungen in den jeweiligen Randbezirken räumlich korreliert sind, also Bezirke am Rand von Berlin vergleichsweise hohe Anteile an Wald- und Landwirtschaftsflächen aufweisen.

Die Kapitalisierung der Landnutzung in den Immobilienpreisen kann auch anhand der impliziten Preise sichtbar gemacht werden (vgl. Tabelle 4). Der implizite Preis wird dabei aus den in der Tabelle 3 errechneten Koeffizienten $(\beta)$ und dem Median $(\tilde{X})$ der jeweiligen Variablen $c$ nach der Formel

$\beta_{c}+2 * \beta_{c^{2}} * \tilde{X}_{c}$

abgeleitet und gibt an, wie hoch der durchschnittliche Preisanstieg bei einer Änderung der erklärenden Variablen (z. B. Grünflächenanteil) wäre. Zur Berechnung wird dabei der durchschnittliche Kaufpreis aller Hausverkäufe in Berlin von 314.088,20 Euro herangezogen. Eine Erhöhung der Grundstücksfläche um einen Quadratmeter würde beispielsweise den Immobilienpreis um 100,26 Euro (0,03\%) heben. Ein Anstieg des Alters der Immobilie um ein Jahr führt zu Preisabschlägen von durchschnittlich 2.125,75 Euro $(-0,68 \%)$. Würde die Abdeckung mit Grünflächen im 500m-Radius um die Immobilie um $1 \%$ erhöht werden, würde dies zu einem durchschnittlichen Preisanstieg der Immobilie von 410,20 Euro $(0,13 \%)$ nach sich ziehen. Dies zeigt, dass eine höhere Zahlungsbereitschaft für sol- 
Abbildung 3 Verteilung der durchschnittlichen Quadratmeterpreise (Grundstücksfläche) des Hauspreissamples über die Berliner Stadtbezirke Quelle: Gutachterausschuss für Grundstückswerte Berlin, Zeitraum 1996-2012

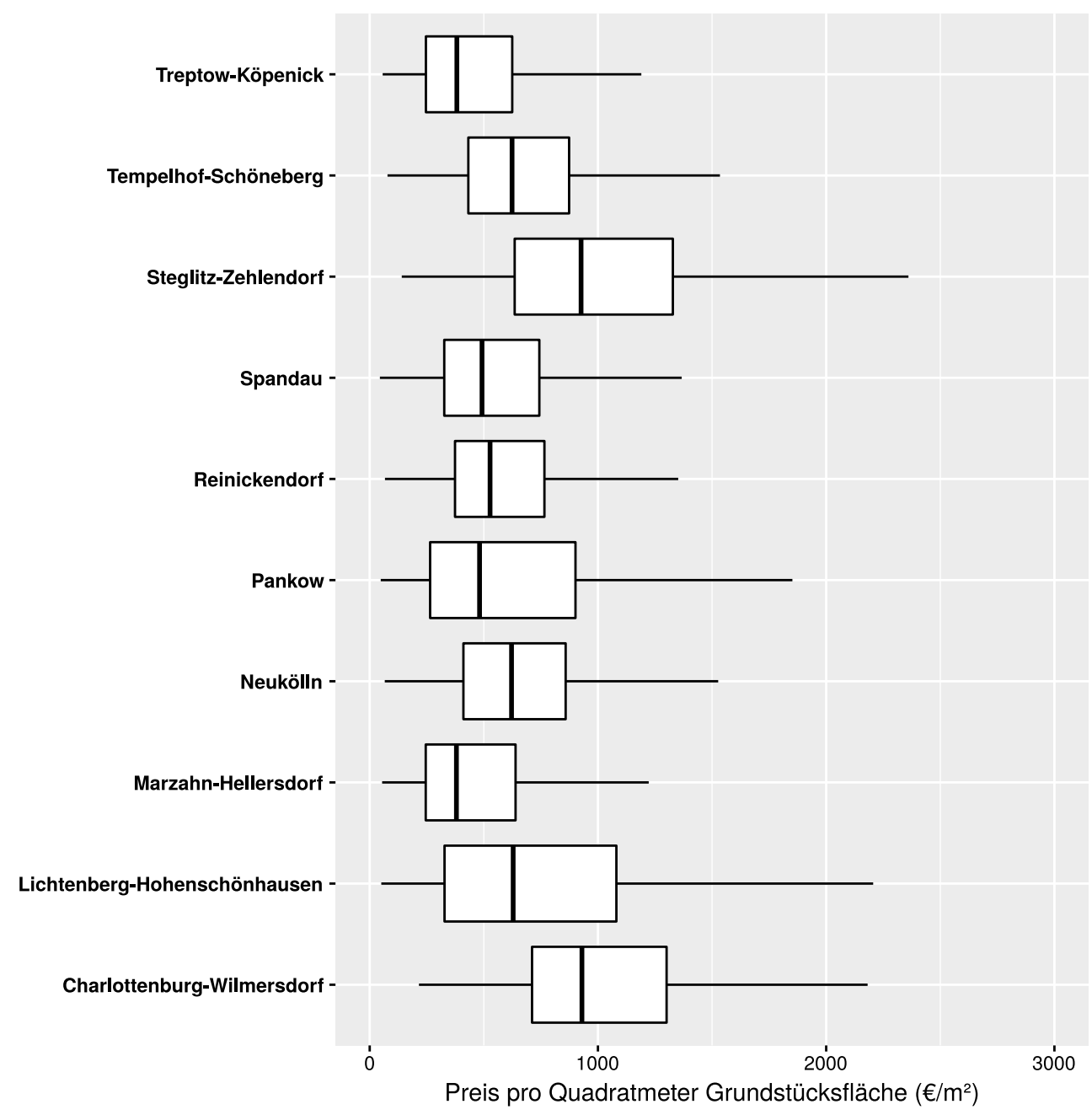

che Immobilien besteht, die mit einem höheren Anteil an Grünflächen umgeben sind. Eine Erhöhung des Anteils an Brachflächen im Umkreis von 500m um die Immobilie würde zu Preisabschlägen von 6.909,94 Euro (-2,2 \%) führen.

\section{Fazit und Ausblick}

Die hier vorgestellten Analysen zeigen deutlich, dass urbane Grünflächen in den Immobilienpreisen kapitalisiert sind, städtisches Grün aber nur ein Einflussfaktor unter vielen ist und im Vergleich zu den strukturellen Daten zum Teil einen eher geringen Einfluss auf die Preise von Immobilien ausübt. Die hier vorgestellten Ergebnisse zur Höhe des Einflusses von Grün auf die Immobilienpreise liegen im Bereich vergleichbarer Analysen zur Wertschätzung von städtischem Grün mittels hedonischer Preismethode (u. a. Tyrväinen/Miettinen 2000: 215; Morancho 2003: 38; Kong/Yin/Nakagoshi 2007: 249). Dabei sollte auch nicht vergessen werden, dass eine Vielzahl weiterer Ökosystemleistungen urbaner Grünflächen eine ökonomische Relevanz 
besitzen (z. B. Klimaschutz, Luftreinhaltung), der Nutzen der Grünanlagen im Rahmen der Immobilienwertmethode also wahrscheinlich deutlich unterschätzt wird.

Die hier gezeigten Ergebnisse werfen auch die Frage nach den stadtökonomischen Konsequenzen eines nachgewiesenen Preiseinflusses von Grünflächen auf, da sich zumindest die Nutzen öffentlicher Grünflächen dabei in Immobilien kapitalisieren, die sich zumeist in privater Hand befinden. Bei Neuentwicklungen von Baugebieten kann über das bauplanungsrechtliche Instrumentarium aus diesem Grund auch der Flächenerwerb und die Anlage von Grünflächen auf die Erwerber des Nettobaulands umgelegt werden. Im Bestand sollte die Instandhaltung der Grünflächen über die Grundsteuer abgedeckt sein, allerdings ist diese jedoch seit Jahren überarbeitungsbedürftig (Wüstemann/Kolbe/von Malottki et al. 2016).

Zudem legen die Ergebnisse zur Kapitalisierung von Grünflächen in Immobilienpreisen nahe, dass Menschen mit vergleichsweise hohem Einkommen einen besseren Zugang zu urbanem Grün haben, da ja Immobilien in unmittelbarer Nähe zu Grünflächen zumeist teurer sind. Dies wird in der Literatur unter Umweltgerechtigkeit diskutiert (Maschewsky 2001; Schröder-Bäck 2012). Erste empirische Untersuchungen in diesem Bereich bestätigen die Annahme, dass der Zugang zu Grünflächen in Deutschland ungleich verteilt ist (Kabisch/Haase 2014; Wüstemann/ Kalisch/Kolbe 2016). Allerdings ist das Wissen in diesem Bereich noch sehr lückenhaft, auch weil aussagekräftige Indikatoren zum Zugang zu städtischem Grün und ein diesbezügliches Monitoring in Deutschland bisher fehlen (Wüstemann/Kolbe/von Malottki et al. 2016).

\section{Literatur}

Acharya, G.; Bennett, L. L. (2001): Valuing Open Space and LandUse Patterns in Urban Watersheds. In: The Journal of Real Estate Finance and Economics 22, 2, 221-237.

Ahlfeldt, G. M.; Maennig, W. (2010a): Impact of sports arenas on land values: evidence from Berlin. In: The Annals of Regional Science 44, 2, 205-227.

Ahlfeldt, G. M.; Maennig, W. (2010b): Substitutability and Complementarity of Urban Amenities: External Effects of Built Heritage in Berlin. In: Real Estate Economics 38, 2, 285-323.

Appelbaum, E. (1979): On the choice of functional forms. In: International Economic Review 20, 2, 449-458.

Bateman, I. (1993): Evaluation of the environment: A survey of revealed preference techniques. Norwich. = CSERGE Working Paper GEC 93-06.

BMUB - Bundesministerium für Umwelt, Naturschutz, Bau und Reaktorsicherheit (2007): Nationale Strategie zur biologischen Vielfalt. Berlin.

Brandt, S.; Maennig, W.; Richter, F. (2013): Do places of worship affect housing prices? Evidence from Germany. Hamburg. = Hamburg Contemporary Economic Discussions 48.

EEA - European Environment Agency (2011): Mapping Guide for a European Urban Atlas. https://cws-download.eea.europa.eu/ local/ua2006/Urban_Atlas_2006_mapping_guide_v2_final.pdf (19.12.2016).
Goh, Y. M.; Costello, G.; Schwann, G. (2012): Accuracy and Robustness of House Price Index Methods. In: Housing Studies 27, 5, 643-666.

Irwin, E. G. (2002): The Effects of Open Space on Residential Property Values. In: Land Economics 78, 4, 465-480.

Kabisch, N.; Haase, D. (2014): Green justice or just green? Provision of urban green spaces in Berlin, Germany. In: Landscape and Urban Planning 122, 129-139.

Kholodilin, K. A.; Mense, A. (2012): Internet-Based Hedonic Indices of Rents and Prices for Flats. Example of Berlin. Berlin. = DIW Discussion Papers 1191.

Kitchen, J. W.; Hendon, W. S. (1967): Land Values Adjacent to an Urban Neighborhood Park. In: Land Economics 43, 3, 357-360.

Kong, F.; Yin, H.; Nakagoshi, N. (2007): Using GIS and landscape metrics in the hedonic price modeling of the amenity value of urban green space: A case study in Jinan City, China. In: Landscape and Urban Planning 79, 3-4, 240-252.

Kuhn, I.; Brandl, R.; Klotz, S. (2004): The flora of German cities is naturally species rich. In: Evolutionary Ecology Research 6, 5, 749-764.

Lutzenhiser, M.; Netusil, N. R. (2001): The effect of open spaces on a home's sale price. In: Contemporary Economic Policy 19, 3, 291-298.

Malpezzi, S. (2003): Hedonic Pricing Models. A Selective and Applied Review. In: O’Sullivan, A.; Gibb, K. (Hrsg.): Housing Economics and Public Policy. Oxford, 67-89.

Maschewsky, W. (2001): Umweltgerechtigkeit, Public Health und soziale Stadt. Frankfurt am Main.

Melichar, J.; Vojáček, O.; Rieger, P.; Jedlička, K. (2009): Measuring the Value of Urban Forest using the Hedonic Price Approach. In: regionální studia I Czech Regional Studies 2, 13-20.

Mense, A.; Kholodilin, K. A. (2014): Noise expectations and house prices: the reaction of property prices to an airport expansion. In: The Annals of Regional Science 52, 3, 763-797.

Morancho, A. B. (2003): A hedonic valuation of urban green areas. In: Landscape and Urban Planning 66, 1, 35-41.

Moulton, B. R. (1986): Random group effects and the precision of regression estimates. In: Journal of Econometrics 32, 3, 385-397.

Moulton, B. R. (1990): An Illustration of a Pitfall in Estimating the Effects of Aggregate Variables on Micro Units. In: The Review of Economics and Statistics 72, 2, 334-338.

Nowak, D. J.; Crane, D. E.; Stevens, J. C. (2006): Air pollution removal by urban trees and shrubs in the United States. In: Urban Forestry \& Urban Greening 4, 3-4, 115-123.

Panduro, T. E.; Lausted Veie, K. (2013): Classification and valuation of urban green spaces - A hedonic house price valuation. In: Landscape and Urban Planning 120, 119-128.

Promann, J. (2012): Die Berücksichtigung des Wohnwertmerkmals Lage in den Mietspiegeln der deutschen Großstädte. Bestandsaufnahme, theoretische Einbettung und ein GIS-gestuitztes Verfahren zur standardisierten Wohnlageermittlung. Lohmar. = Industrieökonomik 8.

Rowntree, R. A.; Nowak, D. J. (1991): Quantifying the role of urban forests in removing atmospheric carbon dioxide. In: Journal of Arboriculture 17, 10, 269-275.

Schröder-Bäck, P. (2012): Ethische Kriterien der Gerechtigkeit für den Zusammenhang von Umwelt und Gesundheit. In: Bolte, G.; Bunge, C.; Hornberg, C.; Köckler, H.; Mielck, A. (Hrsg.): Umweltgerechtigkeit: Chancengleichheit bei Umwelt und Gesundheit. Konzepte, Datenlage und Handlungsperspektiven. Bern, 51-60.

Schulz, R.; Werwatz, A. (2011): Is there an equilibrating relationship between house prices and replacement cost? Empirical evidence from Berlin. In: Journal of Urban Economics 69, 3, 288-302.

Tyrväinen, L.; Miettinen, A. (2000): Property Prices and Urban Forest Amenities. In: Journal of Environmental Economics and Management 39, 2, 205-223. 
Vanslembrouck, I.; Van Huylenbroeck, G.; Van Meensel, J. (2005): Impact of Agriculture on Rural Tourism: A Hedonic Pricing Approach. In: Journal of Agricultural Economics 56, 1, 17-30.

Wüstemann, H.; Kalisch, D.; Kolbe, J. (2016): Towards a national indicator for urban green space provision and environmental ine- qualities in Germany: Method and findings. Berlin. = SFB 649 Discussion Paper 2016-022.

Wüstemann, H.; Kolbe, J.; von Malottki, C.; Vaché, M. (2016): Stadtgrün und Immobilienwerte. In: Kowarik, I.; Bartz, R.; Brenck, M. (Hrsg.): Ökosystemleistungen in der Stadt. Gesundheit schützen und Lebensqualität erhöhen. Berlin/Leipzig, 197-202. 